\title{
Association of postoperative biomarker response with recurrence and survival in patients with hepatocellular carcinoma and high alpha-fetoprotein expressions (> $400 \mathrm{ng} / \mathrm{mL}$ )
}

\author{
Ming-Da WANG ${ }^{1}$, Lei LIANG ${ }^{2}$, Cheng-Wu ZHANG ${ }^{2}$, Wan Yee LAU ${ }^{1,3}$, Feng SHEN ${ }^{1}$, Timothy M. PAWLIK ${ }^{4}$, Dong-Sheng HUANG ${ }^{2}$, Tian YANG ${ }^{* 1,2}$ \\ 'Department of Hepatobiliary Surgery, Eastern Hepatobiliary Surgery Hospital, Second Military Medical University (Navy Medical University), Shanghai, China \\ ${ }^{2}$ Department of Hepatobiliary, Pancreatic and Minimal Invasive Surgery, Zhejiang Provincial People's Hospital, \\ People's Hospital of Hangzhou Medical College, Hangzhou, China \\ ${ }^{3}$ Faculty of Medicine, The Chinese University of Hong Kong, Shatin, New Territories, Hong Kong, China \\ ${ }^{4}$ Department of Surgery, Ohio State University, Wexner Medical Center, Columbus, OH, USA
}

Introduction: High alpha-fetoprotein (AFP) expressions (> $400 \mathrm{ng} / \mathrm{mL}$ ) are associated with poor oncological characteristics for hepatocellular carcinoma (HCC). However, prognosis after liver resection for high-AFP HCC is poorly studied. To investigate long-term recurrence and survival after hepatectomy for high-AFP HCC, and to identify the predictive value of postoperative incomplete biomarker response (IBR) on overall survival (OS) and recurrence-free survival (RFS).

Methods: Patients undergoing curative resection for high-AFP HCC were analyzed. According to the decline magnitude of serum AFP as measured at first follow-up (4-6 weeks after surgery), all patients were divided into the complete biomarker response (CBR) and IBR groups. Characteristics, recurrence, and survival rates were compared.

Results: Among 549 patients, the overall and early recurrence rates in patients with IBR were significantly higher than patients with CBR $(97.8 \%$ vs. $56.4 \%$, and $92.5 \%$ vs. $33.3 \%$, both $p<0.001)$. On multivariate analysis, postoperative IBR was the strongest risk factor with the highest hazard ratio in predicting poor OS $(2.97 ; 2.49-3.45 ; p<0.001)$ and RFS $(4.29 ; 3.31-5.55 ; p<0.001)$.

Conclusions: Postoperative biomarker response of serum AFP can be used in predicting recurrence and survival for high-AFP HCC patients. Once postoperative IBR was identified at first follow-up, subsequent enhanced recurrence surveillance and available treatments against recurrence should actively be considered. 